\title{
Relation between ice sheet internal radio-echo reflections and ice fabric at Mizuho Station, Antarctica
}

\author{
Shuji Fujita ANd Shinji Mae \\ Department of Applied Physics, Faculty of Engineering, Hokkaido University, Kita-13, Nishi-8, Kita-ku, Sapporo 060, Japan
}

\begin{abstract}
The relationship between ice fabric and the internal radio-echo reflections was investigated using observation data collected at Mizuho Station, Antarctica. The data were obtained by $179 \mathrm{MHz}$ radio-echo sounding and the ice fabric was measured from $700 \mathrm{~m}$ Mizuho ice core. The dielectric permittivity tensor at given depths in the ice sheet was calculated from the ice fabric.

The calculated dielectric permittivity tensor showed that the ice sheet at Mizuho Station is a uniaxially birefringent medium. The symmetrical axis of rotation was the same as the flow line. In such a medium, theory predicts that the electric field vectors are allowed only in the two directions parallel and perpendicular to the flow line. The prediction coincided well with the observation: a strong signal was observed only when the transmitting antenna and the receiving antenna, kept parallel to one another, were oriented parallel or perpendicular to the flow line. However, the observed signal strength in these two directions differed from one another at each depth.

It is also shown that the power reflection coefficient due to the variation of ice fabric with depth is of approximately the same order as that due to the density change and is large enough to produce the predominant internal radio-echo reflections.
\end{abstract}

\section{INTRODUCTION}

Yoshida and others (1987) carried out $179 \mathrm{MHz}$ radioecho sounding at Mizuho Station $\left(70^{\circ} 42^{\prime} \mathrm{S}, 44^{\circ} 20^{\prime} \mathrm{E}\right.$, $2230 \mathrm{~m}$ a.s.l.), East Antarctica, in order to detect the birefringence of the ice sheet. They obtained the result that the echo strength of the internal reflections increased when the two parallel antennae azimuths were parallel or perpendicular to the observed flowline, as shown in Figure 1, at depths between 200 and $1300 \mathrm{~m}$. Since the

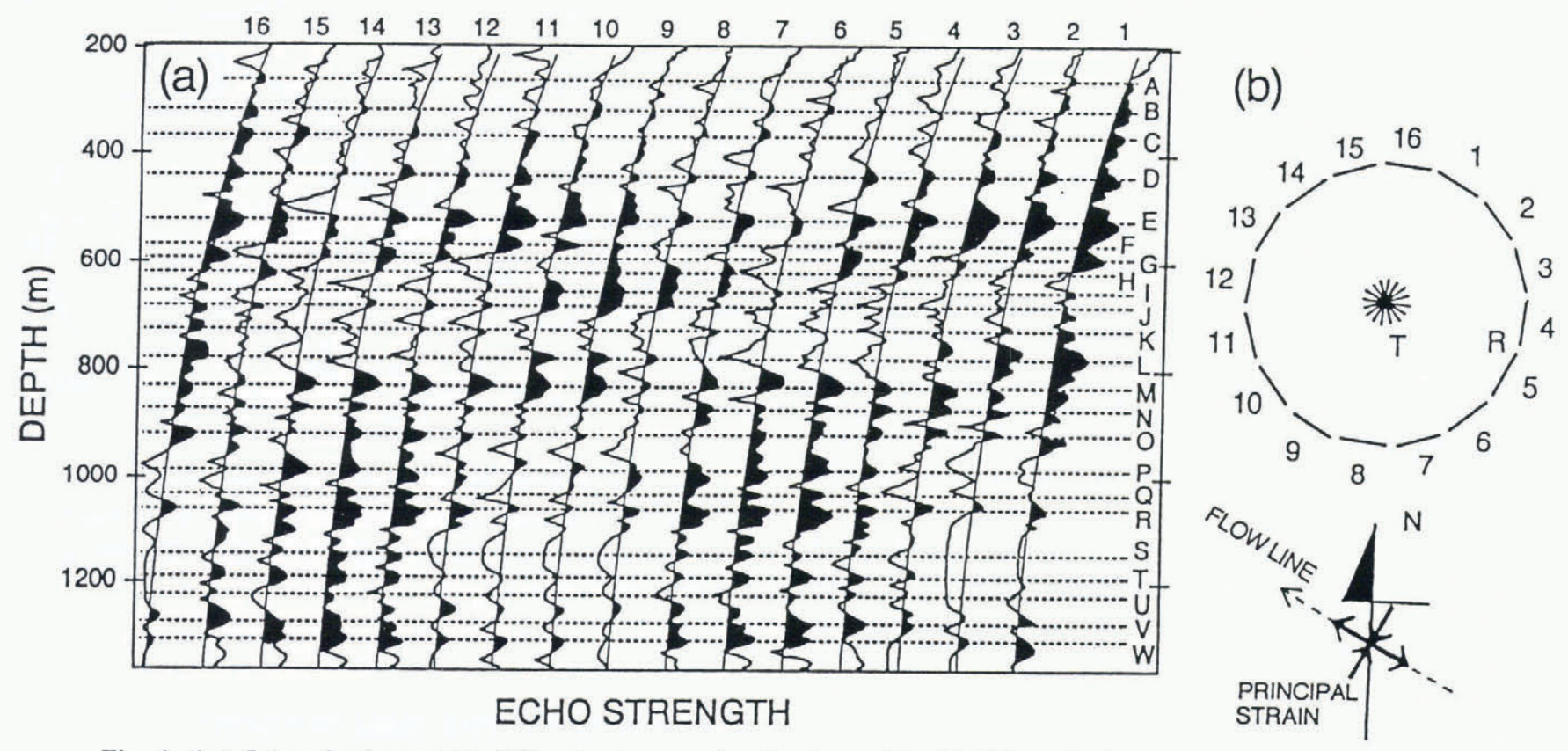

Fig. 1. (a) Internal echoes at 16 different antenna azimuths observed by Yoshida and others (1987) as A-scope data. Echo strengths above the average level of the 16 data sets are dark. (b) $R$ and $T$ represent the two parallel antennae, receiving and transmitting aerials, respectively. The flow line (Nishio and others, 1989) and the axes of the principal strain (Naruse and Shimizu, 1978) are also shown. 
flow line was parallel to the tensile principal strain, it was suggested that the ice fabric, the spatial distribution of the $c$ axis of ice grains, might determine the birefringence of the ice sheet.

Fujita and others (1987) measured the depth profile of ice fabric using $700 \mathrm{~m}$ Mizuho core drilled at Mizuho Station. They showed that the $c$ axes of the grains tended to orient perpendicular to the flow line and that the $c$ axes concentrated gradually on a girdle with increasing depth.

Fujita and others (1993) investigated the dielectric anisotropy of a single crystal of ice at $9.7 \mathrm{GHz}$. It was found that the permittivity parallel to the $c$ axis, $\epsilon_{\| c}^{\prime}$, is larger than $\epsilon_{\perp c}^{\prime}$, perpendicular to the $c$ axis, and that the dieletric anistropy, $\epsilon_{\| c}^{\prime}-\epsilon_{\perp c}^{\prime}$, is 0.037 ( \pm 0.007$)$. This value was constant in the temperature range of the cryosphere and valid over the frequency range from radio wave to microwave. Estimating the power reflection coefficient due to the dielectric anisotropy, Fujita and others (1993) proposed that the internal reflection observed by radio-echo sounding (RES) is caused by the variation of the ice fabric with depth.

In this paper, we discuss the propagation of polarized radio wave in the ice sheet using the ice fabric data of the $700 \mathrm{~m}$ Mizuho core and compare it with the $179 \mathrm{MHz}$ RES data. Then the power reflection coefficient due to variation of ice fabric with depth is also estimated.

\section{RESULT OF RADIO-ECHO SOUNDING AT MIZUHO STATION}

In 1984 Yoshida and others (1987) carried out $179 \mathrm{MHz}$ radio-echo sounding at Mizuho Station. The sounding site was about $1 \mathrm{~km}$ downwards along the flowline from the drilling hole in the Mizuho Station. The radar system was described in detail by Yoshida and others (1987) as follows: six-element Yagi antenna which is linearly polarized transmits a $1.5 \mathrm{~kW}$ (peak) pulse wave in a repetition frequency of $1 \mathrm{kHz}$, and the returned signal is received by the same type of aerial as the transmitting one. The transmitting antenna and receiving antenna were kept parallel to one another at a distance of $3 \mathrm{~m}$, rotating the receiving antenna round the transmitting antenna. Measurements were repeated every $22.5^{\circ}$ intervals of rotation. Finally the internal echoes of 16 different azimuth were obtained. Figure 1(a) shows the observed echoes. Figure 1(b) shows the antenna azimuth at each observation. The directions of the observed flowline (Nishio and others, 1989) and principal strain (Naruse and Shimizu, 1978) are also shown in Figure 1(b). The direction of Nos 1 and 9 is parallel to the flowline. The direction of Nos 5 and 13 is perpendicular to the flowline.

Figure 2 shows the relation between the antenna azimuth and the maximum echo strength at depth denoted by the letters in Figure $1(\mathrm{a})$. The internal echoes at depths between 200 and $800 \mathrm{~m}$ are strongest when two parallel antennae are parallel to the flowline (antennae azimuth Nos 1, 2, 8 and 9, 10, 16 in Figure 1). At depths between 800 and $1300 \mathrm{~m}$, internal echoes are strongest when two parallel antennae are perpendicular to the flowline (antennae azimuth Nos 4, 5, 6 and 12, 13, 14 in Figure 1).

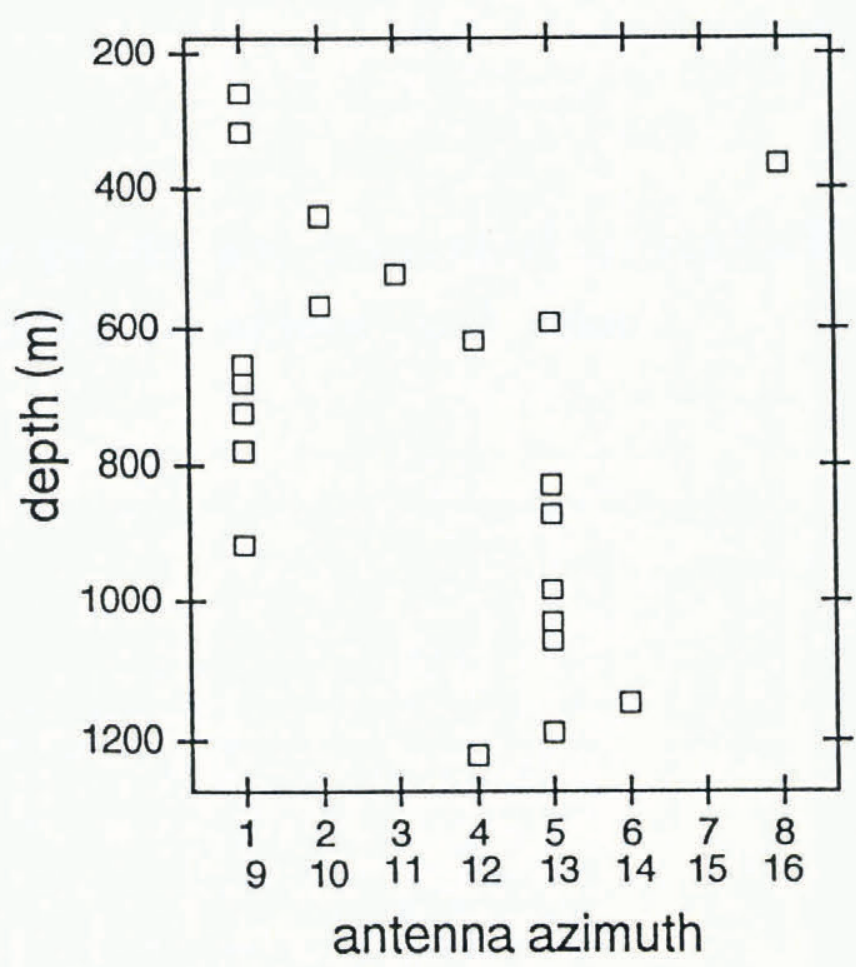

Fig. 2. Antenna azimuth in which the maximum radio-echo strength was observed at each internal reflection. The investigated 23 internal reflections are denoted in Fig. 1(a) by the letters. Antenna azimuth is shown in Fig. 1(b).

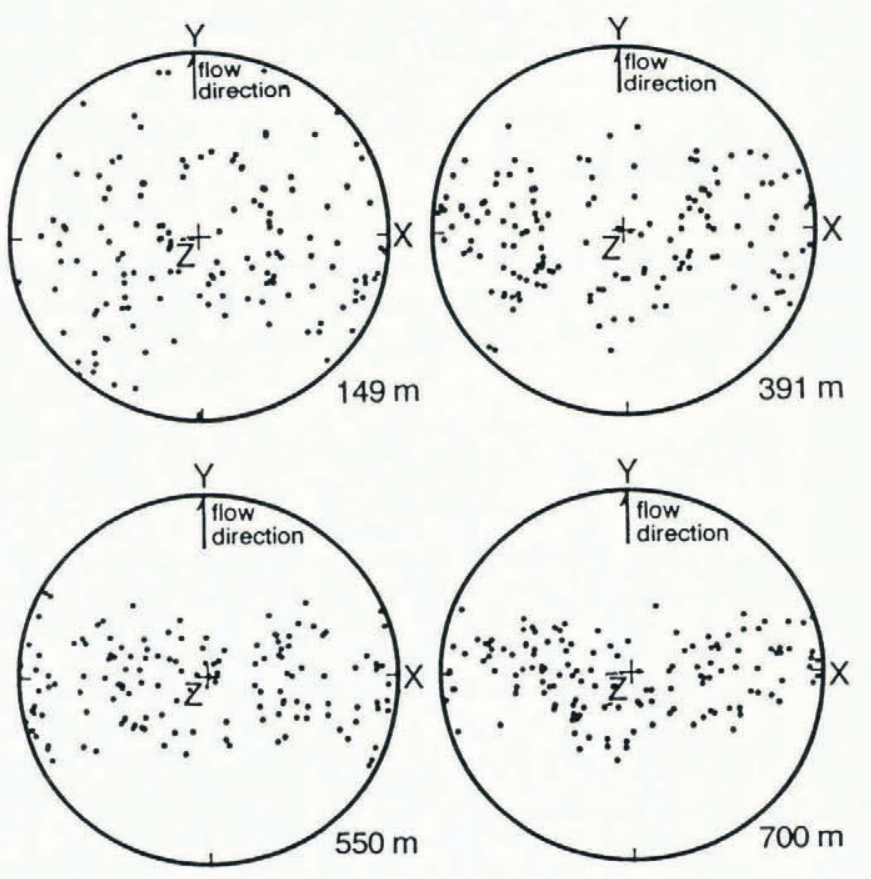

Fig. 3. Typical ice fabric found in the $700 \mathrm{~m}$ Mizuho core. The $c$ axes of crystal grains were concentrated nearly in the vertical plane ( $x-z$ plane) normal to the flow direction ( $y$ axis) of the ice sheet.

\section{ICE FABRIC OF THE MIZUHO CORE}

Ice fabric of the Mizuho core was reported by Narita and others (1986) and Fujita and others (1987). Figure 3 shows the examples of the ice fabric of the Mizuho core at 
four depths. The $c$ axes were in the girdle perpendicular to the flowline. This feature is more pronounced as the depth increases. Fujita and others (1987) found that this concentration was due to tensile strain along the flowline.

\section{ANISOTROPY OF DIELECTRIC PERMITTIVITY TENSOR}

We calculate the dielectric permittivity tensor of the ice sheet at Mizuho Station from ice fabric data. Fujita and others (1993) measured the dielectric permittivity of ice parallel to the $c$ axis $\left(\epsilon_{\| c}^{\prime}\right)$ and perpendicular to the $c$ axis $\left(\epsilon_{\perp c}^{\prime}\right)$ at $9.7 \mathrm{GHz}$ at temperatures between $-32.5^{\circ}$ and $2.5^{\circ} \mathrm{C}$. $\epsilon_{\| c}^{\prime}$ and $\epsilon_{\perp c}^{\prime}$ are given by following equations:

$$
\begin{aligned}
& \epsilon_{\| c}^{\prime}=3.189( \pm 0.006)+0.00092( \pm 0.00007) T \\
& \epsilon_{\perp c}^{\prime}=3.152( \pm 0.003)+0.00086( \pm 0.00005) T
\end{aligned}
$$

where $T$ is the temperature expressed in ${ }^{\circ} \mathrm{C}$. The dielectric anisotropy $\Delta \epsilon^{\prime}, \epsilon_{\| c}^{\prime}-\epsilon_{\perp c}^{\prime}$ is

$$
\Delta \epsilon^{\prime}=0.037( \pm 0.007)+0.00006( \pm 0.00009) T .
$$

The temperature dependence is negligibly small in the temperature range of the cryosphere. Fujita and others (1993) indicates that in the frequency range of $\mathrm{HF}, \mathrm{VHF}$, and microwave frequencies that $\Delta \epsilon^{\prime}$ is 0.037 .

Hargreaves (1978) obtained the macroscopic dielectric permittivity tensor of the polycrystalline ice from ice fabric data. Applying the dielectric mixture theory (Polder and Santen, 1946) to the mixture of anisotropic dielectrics, he showed that the dielectric permittivity tensor of the polycrystalline is expressed by

$$
\epsilon^{\prime}=\sum_{j=1} f_{j} \epsilon^{(j)}
$$

where $f_{j}$ is the volume fraction of the $j$ th crystal grain and $\epsilon^{(j)}$ is the dielectric permittivity tensor the $j$ th crystal grain. Equation (4) is derived on an assumption that the volume average is over a volume which is small compared with the wavelength but sufficiently large compared with the size of individual crystal grain. The dielectric permittivity tensor $\epsilon_{\mathrm{p}}$ of a crystal grain is given by

$$
\epsilon_{\mathrm{p}}=\left(\begin{array}{ccc}
\epsilon_{\perp c}^{\prime} & 0 & 0 \\
0 & \epsilon_{\perp c}^{\prime} & 0 \\
0 & 0 & \epsilon_{\| c}^{\prime}
\end{array}\right)
$$

when we take the $c$ axis as one of the principal axes.

Using Equations (4) and (5), one can express the dielectric permittivity tensor component parallel to the electric field. If we take the angle between the electric field vector and the $c$ axis of $j$ th grain as $\theta_{j}$, it is written as

$$
\epsilon^{\prime}=\epsilon_{\perp c}^{\prime}+\sum_{j=1}^{N} f_{j} \Delta \epsilon^{\prime} \cos \theta_{j}
$$

where $N$ is the total number of the crystal grains in the ice medium. For simplicity, we approximate that the volume of all grains is the same, then Equation (6) is rewritten by

$$
\epsilon^{\prime}=\epsilon_{\perp c}^{\prime}+\Delta \epsilon^{\prime} D_{\mathrm{a}}
$$

$$
D_{\mathrm{a}}=\frac{1}{N} \sum_{j=1}^{N} \cos \theta_{j} .
$$

Here $D_{\mathrm{a}}$ is a factor which expresses the degree of contribution of $\Delta \epsilon^{\prime}$ to the component of dielectric permittivity tensor.

Ice fabric of the Mizhuho core has a clear girdle pattern as shown in Figure 3. This pattern indicates that the dielectric permittivity tensor of the Mizuho core has a uniaxial symmetry and its axis of symmetry is parallel to the flowline. Using ice fabirc, $D_{\mathrm{a}}$ is calculated along three axes: the axis along the flowline ( $y$ axis), the axis along the transverse direction ( $x$ axis) and the axis along the vertical ( $z$ axis). The calculated results appear in Figure 4 , which shows that the $D_{\mathrm{a}}$ of the $y$ axis is smaller than those of the $x$ and $z$ axes, and that the difference increases with depth. Therefore we can consider that $D_{\mathrm{a}}$ has approximately a uniaxial symmetry.

\section{WAVE PROPAGATION}

Hargreaves (1977) pointed out that when plane-wave propagates in the uniaxially birefringent medium, only two directions of the electric field vector are allowed. When a linearly polarized wave in air is incident

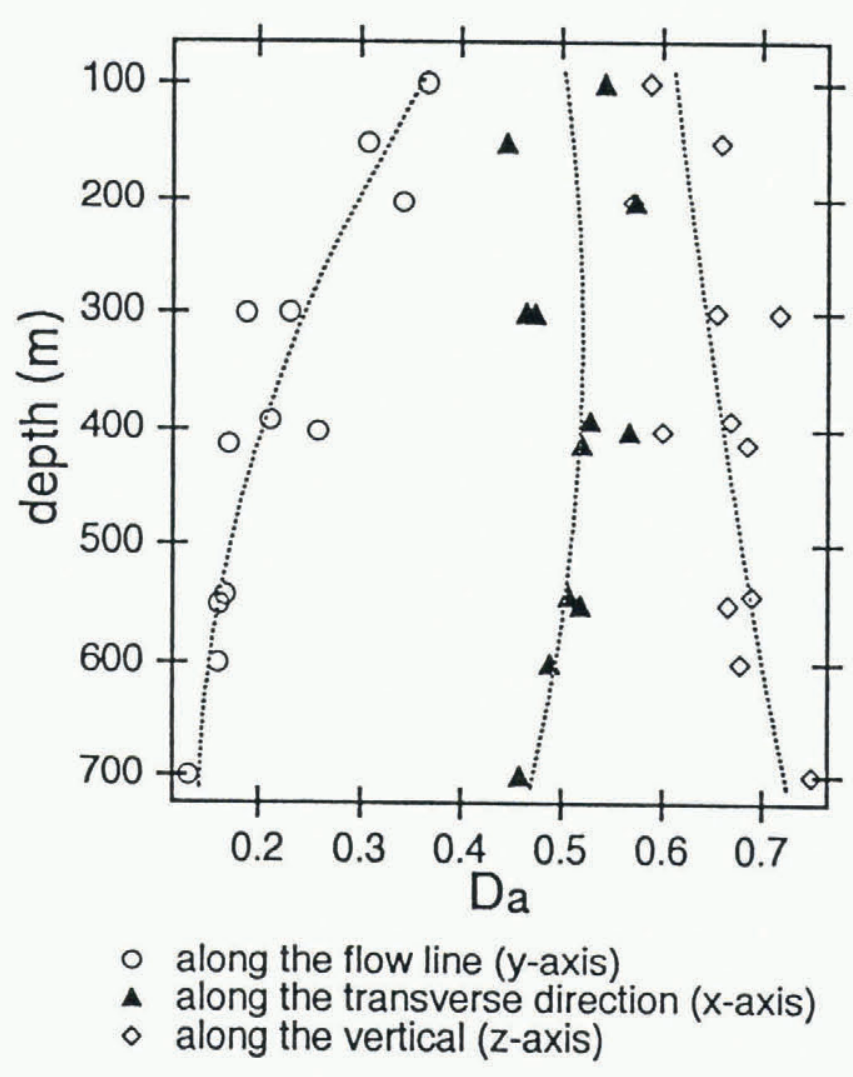

Fig. 4. Contribution of the dielectric anisotropy to each component of the dielectric permittivity tensor calculated from ice fabric data of the $700 \mathrm{~m}$ Mizuho core. $D_{\mathrm{a}}$ is defined in Equations (7) and (8). Calculation of $D_{\mathrm{a}}$ was carried out along the following three axes: the axis along the flowline ( $y$ axis), the axis along the transverse direction ( $x$ axis) and the axis along the vertical ( $z$ axis). 
normally on the medium, the incident electric vector becomes resolved into two components along the directions of allowed electric vectors in the medium. These two directions are uniquely specified by the direction of propagation and the symmetry axis of dielectric permittivity tensor. Initially the two components are in phase, but after passage through the medium, since they have different magnitudes of propagation vector, they are out of phase. When the wave returns from the medium to air, it is elliptically polarized.

As described above, the dielectric permittivity tensor of the ice sheet at Mizuho Station has approximately uniaxial symmetry, that is, ice at the station must be a uniaxially birefringent medium and its axis of symmetry is along the flowline. In the case of Mizuho Station, the electric field vectors must be along the flowline ( $y$ axis) and along the transverse direction ( $x$ axis). If we assume that the components of the dielectric permittivity tensor along the transverse direction ( $x$ axis) and the vertical $(z$ axis) are equal, then the magnitudes of the two propagation vectors along $z$ axis are given by Hargreaves (1977) as

$$
k_{1}^{2}=\left(\omega^{2} / c^{2}\right) \epsilon_{x}: k_{2}^{2}\left(\omega^{2} / c^{2}\right) \epsilon_{y}
$$

where $k_{1}$ and $k_{2}$ are the magnitudes of the propagation vectors of the ordinary wave and the extraordinary wave, respectively. $\epsilon_{x}$ and $\epsilon_{y}$ are the component of the dielectric permittivity tensor along the transverse direction ( $x$ axis) and along the flowline $y$ axis), respectively. $\omega$ is angular frequency. $c$ is the velocity of the electromagnetic waves in free space. One can calculate the phase difference, $\phi$, between the ordinary wave and the extraordinary wave using $k_{1}$ and $k_{2}$ as follows:

$$
\phi=2 \int_{z_{1}}^{0}\left(k_{1}-k_{2}\right) \mathrm{d} z
$$

where $z_{1}$ is the depth of an internal reflection horizon.

We suppose that a linearly polarized transmitting antenna is at an angle $\alpha$ to the $x$ axis. If the receiving antennae are kept parallel to the transmitting one and rotated together, the received signal strength which is reflected from within a uniaxial birefringent medium is given by Hargreaves (1977) as

$E_{\|}^{2}=E_{y}^{2}+\frac{1}{2} \sin ^{2} 2 \alpha\left(E_{x} E_{y} \cos \phi-E_{y}^{2}\right)+\cos ^{4} \alpha\left(E_{x}^{2}-E_{y}^{2}\right)$

Here the transmitted wave is assumed to be $E_{0}(\cos \alpha$, $\sin \alpha, 0)$, and the received wave is

$$
\left\{\begin{array}{l}
x \text { component } E_{x} \cos \alpha \cos (\omega t-k z) \\
y \text { component } E_{y} \sin \alpha \cos (\omega t-k z+\phi) .
\end{array}\right.
$$

$E_{x} \neq E_{y}$ because the transmission, attenuation and reflection coefficient are not equal in these directions. However, becasue we do not know the difference of these coefficients, for simplicity, $E_{x}=E_{y}$ is assumed. And $E_{\|}^{2}$ is estimated as a function of the antennae azimuth $\alpha$. The result of the estimation is shown in Figure 5 which indicates that the return signal becames strongest when the two parallel antennae are oriented parallel to the flowline ( $y$ axis) and parallel to the transverse direction ( $x$ axis).

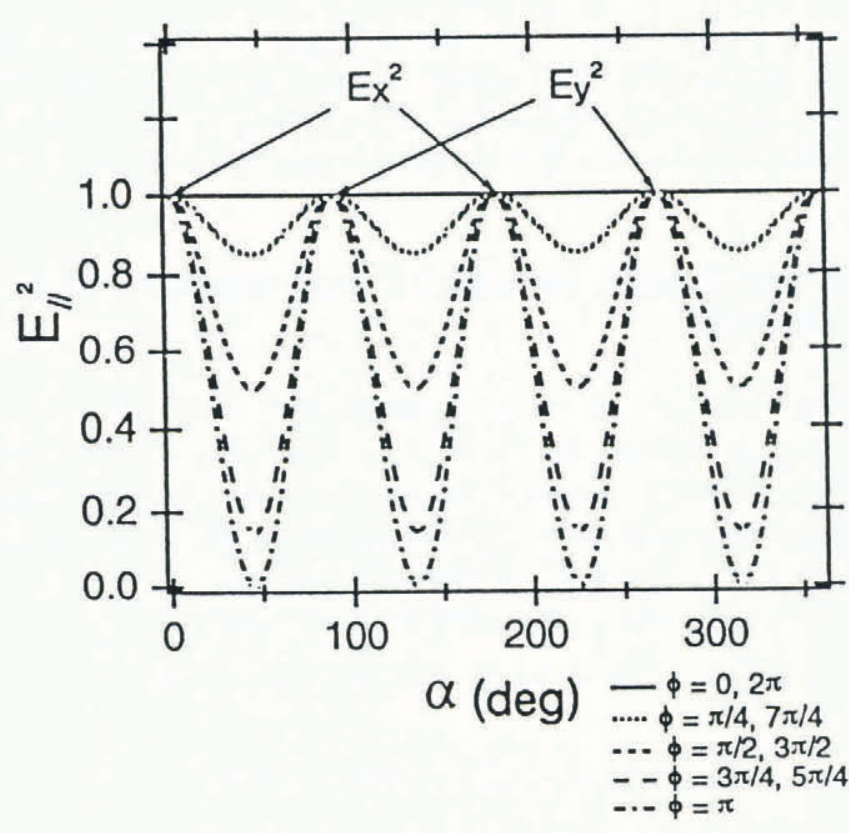

Fig. 5. $E_{\|}^{2}$ as a function of $\alpha$ and $\phi$ calculated with Equation (11). The return signal strength, $E_{\|}^{2}$, is shown as the normalized value. When the transmitting and the receiving antennae were oriented parallel to the $x$ axis or perpendicualr to the $x$ axis, the calculated value became the strongest. $\phi$ is the phase difference between the ordinary and the extraordinary wave. $E_{x}=E_{y}$ is assumed in the calculations. The peaks at $\alpha=0^{\circ}$ and $180^{\circ}$ represent $E_{x}^{2}$. The peaks at $\alpha=90^{\circ}$ and $270^{\circ}$ represent $E_{y}^{2}$.

The actual strength of the radio-echo internal reflections observed by Yoshida and others (1987) were strongest when the antennae were oriented parallel or perpendicular to the flowline as shown in Figure 2. Theoretical prediction on the anisotropy of signal strength can explain the actual observation. At depths between 200 and $800 \mathrm{~m}$, the return signals were strongest when the antennae were oriented parallel to the flowline ( $y$ axis) but weakest when the antennae were oriented perpendicular to the flowline ( $x$ axis) although this feature was not very clear above $600 \mathrm{~m}$. On the other hand, at depths between 800 and $1300 \mathrm{~m}$, the return signals were strongest when the antennae were oriented perpendicular to the flowline ( $x$ axis) but weakest when the antennae were oriented parallel to the flowline ( $y$ axis). In other words, the strong echo appears only in one orientation of the predicted two orientations at each depth. This fact means that although we assumed $E_{x}=E_{y}$ to estimate $E_{\|}^{2}$, it seems that this assumption is not correct. Since $E_{x}$ and $E_{y}$ are affected by the transmission, attenuation and reflection coefficinets, it is difficult to specify which one is the dominant cause of the difference between $E_{x}$ and $E_{y}$. However, as one of the possible causes of difference between $E_{x}$ and $E_{y}$, we propose the attenuation due to volume scattering which occurs due to the difference in dielectric permittivity between adjacent grains at the grain boundaries.

The fact that ice single crystals have dielectric anisotropy means that the dielectric permittivity changes 
at grain boundaries in polycrystalline ice. The maximum difference in dielectric permittivity between adjacent grains os $0.037( \pm 0.007)$. The grain boundary network in polycrystalline ice is also the dielectric permittivity boundary network. In addition, the mean difference of the dielectric permittivity between adjacent grains closely depends on the relative orientation between the electric field vector and the ice fabric. The mean difference, $d$, of the dielectric permittivity between adjacent grains can be calcualted from ice fabric data with the following equation:

$$
d=\frac{1}{(N-1)^{2}} \sum_{i=1}^{N} \sum_{j=1}^{N}\left(\epsilon_{i}-\epsilon_{j}\right)
$$

where $\epsilon_{i}$ and $\epsilon_{j}$ are the components of the dielectric permittivity tensor of $i$ th and $j$ th crystal grains, respectively. If we take the angle between the electric field and the $c$ axis of the $i$ th and $j$ th grains as $\theta_{i}$ and $\theta_{j}$, respectively, Equation (13) becomes

$$
\begin{gathered}
d=\Delta \epsilon^{\prime} D_{\mathrm{b}} \\
D_{\mathrm{b}}=\frac{1}{(N-1)^{2}} \sum_{i=1}^{N} \sum_{j=1}^{N}\left(\cos \theta_{i}-\cos \theta_{j}\right) .
\end{gathered}
$$

$D_{\mathrm{b}}$ expresses the contribution of dielectric anisotropy, $\Delta \epsilon^{\prime}$, to $d$, like $D_{\mathrm{a}}$. If a random fabric pattern is considered, $D_{\mathrm{b}}$ is about 0.34 . Then $d$ is about 0.013 .

The calculated $D_{\mathrm{b}}$ from the fabric data of the Mizuho

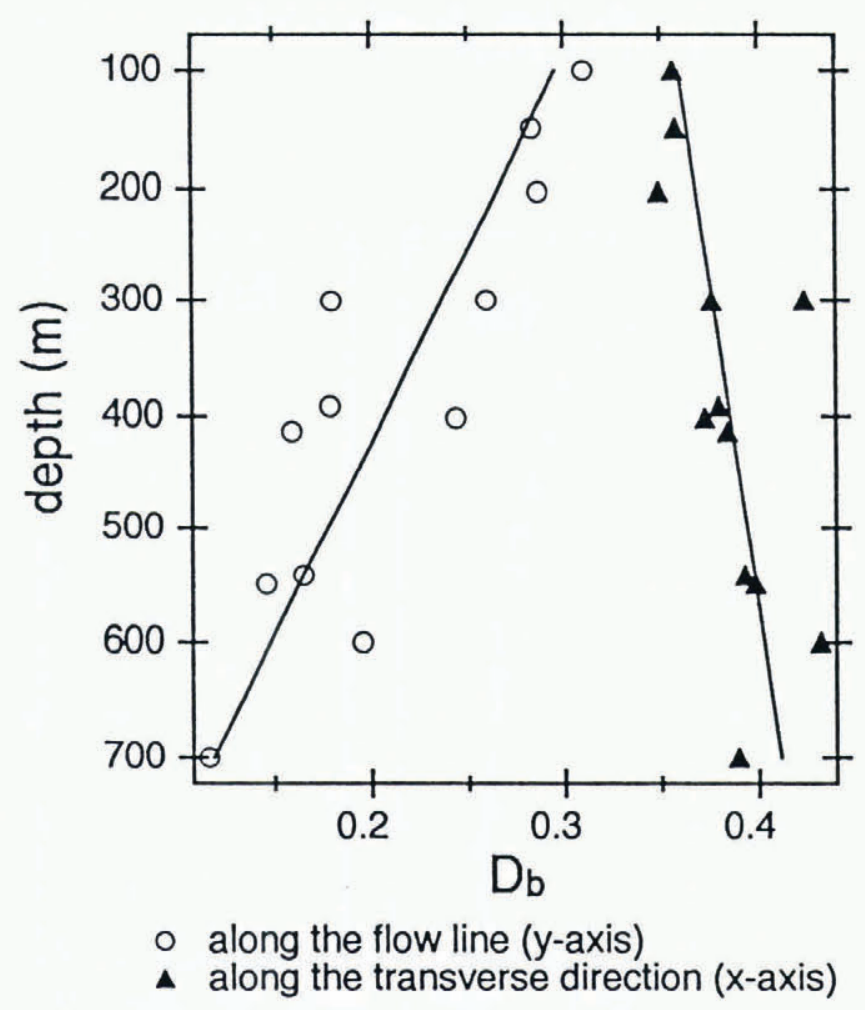

\footnotetext{
Fig. 6. The mean difference of dielectric permittivity between adjacent grains calculated from ice fabric data. $D_{\mathrm{b}}$ is defined in Equations (14) and (15). Calculations were carried out with Equation (15) along the orientation parallel to the flowline ( $y$ axis) and perpendicular to the flowline (x axis).
}

core appears in Figure 6, which shows clearly that $D_{\text {b }}$ perpendicular to the flowline is larger than that parallel to the flowline. The difference between the two $D_{\mathrm{b}}$ increases with depth. It is not clear how much $D_{\text {b }}$ anisotropy contributes to the volume scattering of the electromagnetic waves but if the loss due to volume scattering occurs dominantly when the antennae are oriented perpendicular to the flowline, the observed result can be explained. That is, the scattering loss may weaken the strength of radio-echo in the orientation perpendicular to the flowline at depths between 200 and $800 \mathrm{~m}$.

\section{REFLECTION}

\section{Reflection due to variation of ice fabric}

The reflection of the electromagnetic waves occur due to change in bulk impedance between layers in the ice sheet. So far, several causes of change in impedance has been proposed. They are (1) density fluctuation, (2) conductivity change due to change in acidity and (3) change in ice fabric.

Harrison (1973) proposed a hypothesis that the variation of ice fabric was one of the major causes of internal reflections observed by RES in the ice sheets. Unfortunately it has not been supported mainly because the dielectric anisotropy was not considered to be so large as to produce the internal reflections. However, Fujita and others (1993) showed that dielectric anisotropy is $0.037( \pm 0.07)$. We show here that this value is large enough for small changes in ice fabric to produce detectable internal reflections.

If the reflection of the electromagnetic waves occur due to only permittivity change from $\epsilon^{\prime}$ to $\epsilon^{\prime}+\delta \epsilon^{\prime}$, then the power reflection coefficient, PRC, is given by (Paren and Robin, 1975)

$$
\operatorname{PRC}=\left|\frac{\delta \epsilon^{\prime}}{4 \epsilon^{\prime}}\right| .
$$

When permittivity change is due only to the variation of ice fabric, Equation (16) becomes

$$
\operatorname{PRC}=\left|\frac{\Delta \epsilon^{\prime} \delta D_{\mathrm{a}}}{4 \epsilon^{\prime}}\right|^{2}
$$

where $\delta D_{\mathrm{a}}$ is the change of $D_{\mathrm{a}}$.

Calculated PRCs using Equation (17) and the dielectric permittivity tensor in Figure 4 are shown in Figure 7. In the calculation, the deviation of $D_{\mathrm{a}}$ from fitting curves at each depth was used as $\delta D_{\mathrm{a}} .3 .14$ was used as $\epsilon^{\prime}$, calculated from Equations (1) and (2) with $T=-30^{\circ} \mathrm{C}$, which is roughly the annual mean temperature at Mizuho Station. In this case, PRC along the flowline and along the transverse line is calculated because the electric field vector is allowed only in these two directions, as discussed above. The calculated PRCs means the the PRCs due to variation of the fabric with depth is large enough to produce the detectable internal radio-echo reflections. The largest values of PRC were about $-75 \mathrm{~dB}$. In addition, calculated PRCs also show that PRCs depend on the direction of the electric field vector. 


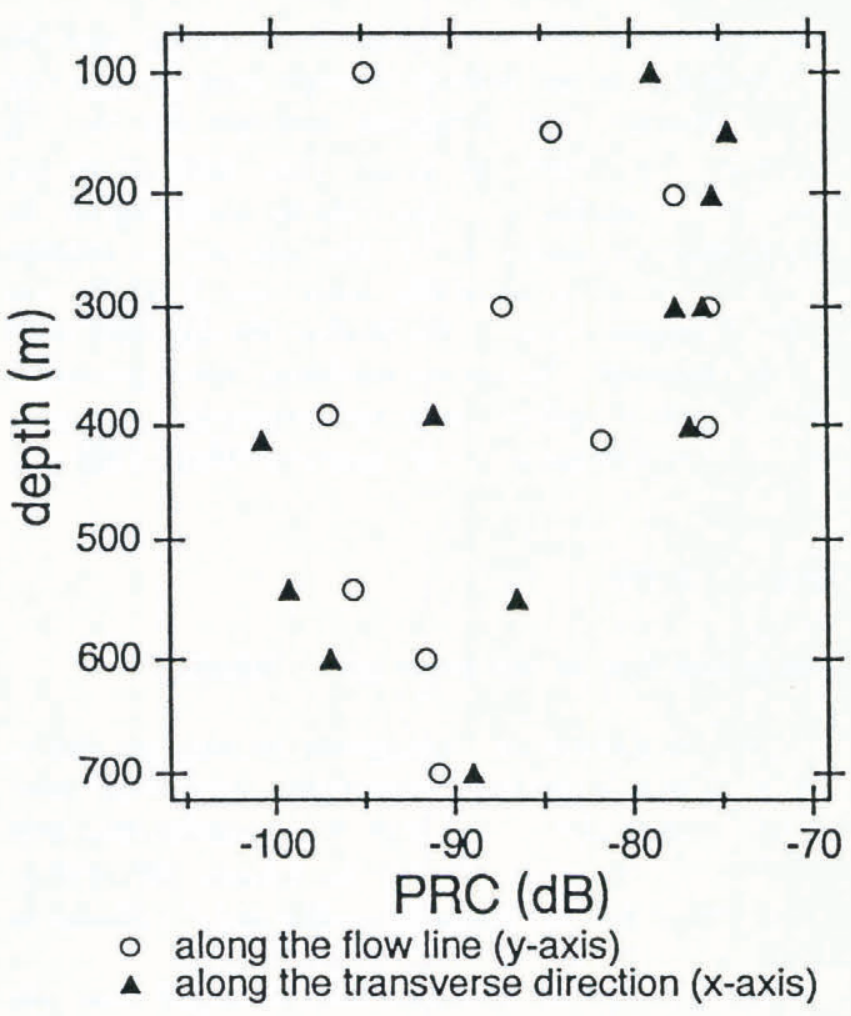

Fig. 7. The power reflection coefficient (PRC) of the radio-echo internal reflections due to variation of ice fabric with depth. The PRCs were calculated with Equation (17) from the dielectric permittivity tensor calculated from ice fabric data.

Although the calculations are based on only sporadic 12 depths in the $700 \mathrm{~m}$ deep core, the calculated PRCs indicate the magnitude of PRCs due to the variation of ice fabric. The variation of fabric in very short distances of depth can occur, for example, between layers which contain volcanic ash and enclosing ice. Such layers are reported by Gow and Williamson (1976) in the Byrd Station core. They investigated the ice fabric of six layers which contain volcanic ash (cloudy bands) and enclosing ice, and found that crystal axes tend to be more tightly clustered about the vertical in the cloudy bands than those in the enclosing ice. In the light of this study, such layers must raise the large value of PRC. In the Mizuho core, only one visible dirt band was found at at a depth of $500.7 \mathrm{~m}$ (Fujii and Watanabe, 1988). This depth coincides with the depth at which the strongest echo was found (Yoshida and others, 1987) at peak ' $E$ ' in Figure $1(\mathrm{a})$.

\section{Reflection due to other causes}

Internal reflections at Mizuho Station must be caused by all or some of the mechanisms shown above. It is unfortunate that ice-fabric data and density data of the Mizuho Station core are based on spot measurements and that there are few conductivity data. As for density data, Nakawo and Narita (1985) give the most accurate data measured with the hydrostatic method. It is shown in Figure 8(a). From Equation (16), PRC due to density variation is given by

$$
\mathrm{PRC}=\left|\frac{\left(\frac{\mathrm{d} \epsilon^{\prime}}{\mathrm{d} \rho}\right) \delta \rho}{4 \epsilon^{\prime}}\right|^{2}
$$

where $\delta \rho$ is the deviation of the density from the fitting curve at each depth expressed by $\mathrm{kg} / \mathrm{m}^{3}$. ( $\left(\frac{\mathrm{d} \epsilon^{\prime}}{\mathrm{d} \rho}\right)$ is 3.08 $\times 10^{-3}\left(\mathrm{~m}^{3} \mathrm{~kg}^{-1}\right)$ (Fujita and others, 1991). The calculated $\delta \rho$ from density data is at most $0.5\left(\mathrm{~kg} / \mathrm{m}^{3}\right)$. Since the experimental error of the density measurement is 1 to $2\left(\mathrm{~kg} / \mathrm{m}^{3}\right.$ ) (Nakawo and Narita, 1985), the calculated $\delta \rho$ from the density data is only fluctuation within the experimental error. But PRCs calculated from this fluctuation can be a measure to estimate the possible maximum PRC by this mechanism. The PRCs calculated using the density data are shown in Figure $8(\mathrm{~b})$. The PRCs range between -70 and $-90 \mathrm{~dB}$. They are almost the same magnitude as the PRCs due to the variation of ice fabric. Since the density variation with depth becomes less at deeper layers, PRC due to the density variation decreases with increasing depth and the other mechanisms become the dominant causes of internal reflections. Compared with the fact that the PRC due to the variation of ice fabric has anisotropy, the PRC due to density variation is isotropic.

Changes in conductivity due to acidity in ice may also be the cause of internal reflections observed at Mizuho Station. This mechanism gives another explanation to the fact that the strongest echo was observed at around $500 \mathrm{~m}$ deep, where visible dirt layers were observed, as pointed out by Yoshida and others (1987). Unfortunately, because the core data is insufficient, it is difficult to discuss the exact cause of each individual internal reflection horizon at present.

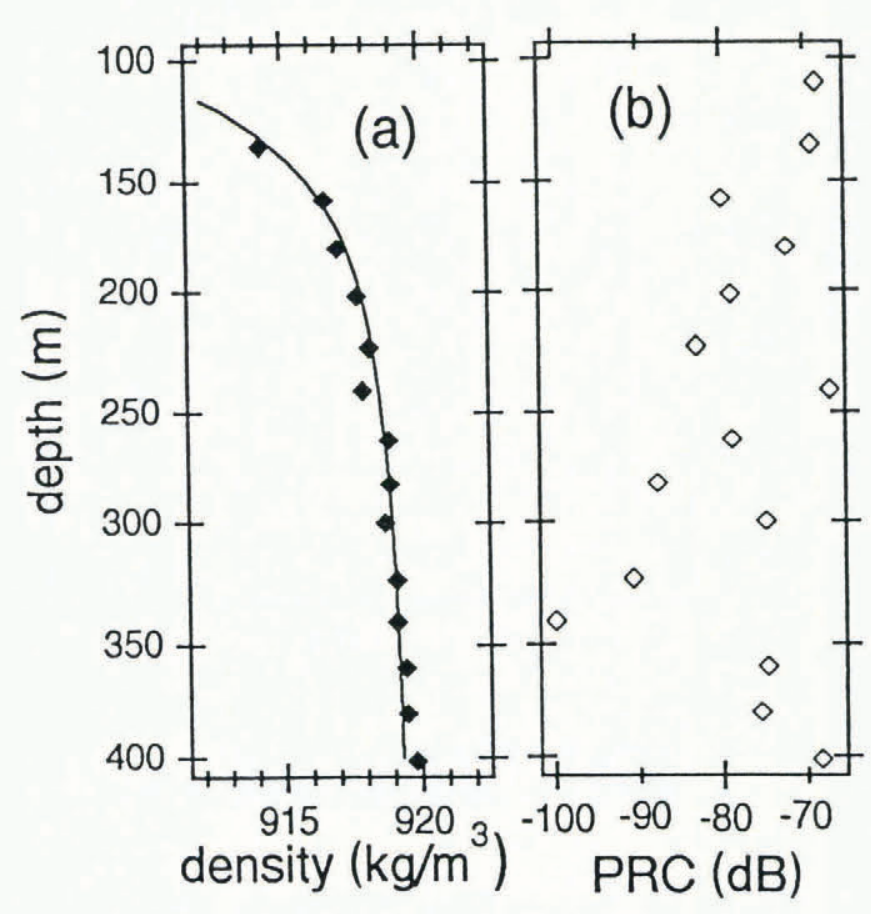

Fig. 8. (a) Depth-density profile of the $700 \mathrm{~m}$ Mizuho core measured with the hydrostatic method (Nakawo and Narita, 1985). (b) The calculated power reflection coefficient (PRC) due to density variation with depth. 
To discuss which mechanism contributes to each reflection, one should investigate the frequency dependence and the temperature dependence of PRC of each reflection horizon, because PRCs due to changes in permittivity and changes in conductivity must have different dependence on frequency and temperature over the frequency range of RES. The natures of PRCs due to different causes will be discussed elsewhere. It should also be investigated how often and how large conductivity and ice fabric can be varied in ice sheets. Ice core analysis is the only way to investigate this. The conductivity profile and ice fabric profile of the ice cores drilled from ice sheets should be compared. The former can be measured with the Dielectric Profiling Technique (Moore and Paren, 1987), but one needs to develop a new technique with which ice fabric profiles can be measured conveniently. These studies are necessary for correct understanding of internal reflection horizons and internal structure of ice sheets.

\section{ACKNOWLEDGEMENT}

The authors thank Dr M. Yoshida at Hakusan Industry for providing the RES data.

\section{REFERENCES}

Fujii, Y. and O. Watanabe. 1988. Microparticle concentration and electrical conductivity of a $700 \mathrm{~m}$ ice core from Mizuho Station, Antarctica. Ann. Glaciol., 10, 38-42.

Fujita, S., M. Nakawo and S. Mae. 1987. Orientation of the 700-m Mizuho core and its strain history. Proceedings of the NIPR Symposium on Polar Meteorology and Glaciology 1, 122-131.

Fujita, S., M. Shiraishi and S. Mae. 1991. Measurement on dielectric constants of polar and artificial ice at $10 \mathrm{GHz}$. In 1991 International Geoscience and Remote Sensing Symposium Digest. Espoo, Helsinki University of Technology, 1567-1570.

Fujita, S., S. Mae and T. Matsuoka. 1993. Dielectric anisotropy in ice Ih at $9.7 \mathrm{GHz}$. Ann. Glaciol., 17 (see paper in this volume).

Gow, A.J. and T. Williamson. 1976. Rheological implications of the internal structure and crystal fabrics of the West Antarctic ice sheet as revealed by deep core drilling at Byrd Station. CRREL Rep. 76-35.

Hargreaves, N. D. 1977. The polarization of radio signals in the radio echo sounding of ice sheets. F. Phys. D. Appl. Phys., 10(9), 1285-1304.

Hargreaves, N.D. 1978. The radio-frequency birefringence of polar ice. 7 . Glaciol., 21 (85), 301-313.

Harrison, C. H. 1973. Radio echo sounding of horizontal layers in ice. 7. Glaciol., 12(66), 383-397.

Moore, J. C. and J. G. Paren. 1987. A new technique for dielectric logging of Antarctic ice cores. 7. Phys. (Paris), 48, Colloq. C1, 155-160. (Supplément au 3.)

Nakawo, M. and H. Narita. 1985. Density profile of a $413.5 \mathrm{~m}$ deep fresh core recovered at Mizuho Station, East Antarctica. Mem. Natl. Inst. Polar Res. Special Issue 39, 141-156.

Narita, H., M. Nakawo and Y. Fujii. 1986. Textures and fabrics of 700-m deep ice core obtained at Mizuho Station, East Antarctica. Mem. Natl. Inst. Polar Res. Special Issue 45, 74-77.

Naruse, R. and H. Shimizu. 1978. Flow line of the ice sheet over Mizuho Plateau. Mem. Natl. Inst. Polar Res. Special Issue 7, 227-234.

Nishio, F., S. Mae, H. Ohmae, S. Takahashi, M. Nakawo and K. Kawada. 1989. Dynamical behavior of the ice sheet in Mizuho Plateau, East Antarctica. Proceedings of the NIPR Symposium on Polar Meteorology and Glaciology 2, 97-104.

Paren, J. G. and G. de Q. Robin. 1975. Internal reflections in polar ice sheets. F. Glaciol., 14(71), 251-259.

Polder, D. and J.H. van Santen. 1946. The effective permeability of mixtures of solids. Physica, 12(5), 257271.

Yoshida, M., K. Yamashita and S. Mae. 1987. Bottom topography and internal layers in East Dronning, Maud Land, East Antarctica, from $179 \mathrm{MHz}$ radio echo-sounding. Ann. Glaciol., 9, 221-224.

The accuracy of references in the text and in this list is the responsibility of the authors, to whom queries should be addressed. 